\title{
Experimental Evidence of Localized Oscillations in the Photosensitive Chlorine Dioxide-Iodine-Malonic Acid Reaction
}

\author{
David G. Míguez, ${ }^{1}$ Sergio Alonso, ${ }^{2}$ Alberto P. Muñuzuri, ${ }^{3}$ and Francesc Sagués ${ }^{4}$ \\ ${ }^{1}$ Department of Systems Biology, Harvard Medical School, 02115 Boston, Massachusetts, USA \\ ${ }^{2}$ Abteilung Physikalische Chemie, Fritz-Haber-Institut der Max-Plank-Gesellschaft, 14195 Berlin, Germany \\ ${ }^{3}$ Grupo de Sistemas Complexos, Facultade de Física, Universidade de Santiago de Compostela, 15782 Santiago de Compostela, Spain \\ ${ }^{4}$ Departament de Química Física, Universitat de Barcelona, 08028 Barcelona, Spain
}

(Received 21 April 2006; published 24 October 2006)

\begin{abstract}
The interaction between Hopf and Turing modes has been the subject of active research in recent years. We present here experimental evidence of the existence of mixed Turing-Hopf modes in a twodimensional system. Using the photosensitive chlorine dioxide-iodine-malonic acid reaction (CDIMA) and external constant background illumination as a control parameter, standing spots oscillating in amplitude and with hexagonal ordering were observed. Numerical simulations in the Lengyel-Epstein model for the CDIMA reaction confirmed the results.
\end{abstract}

DOI: 10.1103/PhysRevLett.97.178301

Pattern formation has become a classical issue of interest in many branches of science ranging from physics, chemistry, and biology to social disciplines or even in relation to some technological applications. Often, patterns are described as a result of symmetry-breaking bifurcations from some featureless state. Two model scenarios in the particular class of reaction-diffusion (RD) systems are the (uniform) Hopf bifurcation to (pure) time-oscillatory regimes and the (stationary) Turing instability to (steady) spatially periodic solutions [1]. Given the wide parameter range that is usually necessary to characterize RD systems, the interest in investigating situations of competing instabilities appears totally justified. In particular the simplest envisaged scenario is that of the interaction between a pair of pure Turing and Hopf modes near a codimension-two bifurcation [2]. Under such conditions, a spatially extended system would be ideally patterned as a lattice, or, more realistically, composing different phase-coherent patches of oscillating Turing motifs. This instability coupling is the issue addressed here both experimentally and by numerical simulations.

Turing-like patterns were first observed in the form of an array of spots or an irregular arrangement of stripes in chemical reactors [3,4]. Quite naturally, thus, and already 15 years ago, the interaction of the Hopf and Turing instabilities was analytically investigated in the LengyelEpstein model of the chlorite-iodide-malonic acid (CIMA) reaction [5] and soon later invoked to interpret peculiar wave phenomena observed in quasi-one-dimensional reactors [6]. Since then the question has been continuously revisited first in relation to RD models $[7,8]$ and more recently in relation to superlattice patterns [9] or segmented waves [10]. Oscillatory Turing patterns have been also evidenced numerically in two coupled layer systems when oscillations occur in one layer and the other supports Turing structures [11]. Different from chemical media, analytical and numerical studies near codimensiontwo Turing-Hopf bifurcations have been applied to models
PACS numbers: $82.40 . \mathrm{Ck}, 47.54 .-\mathrm{r}, 89.75 . \mathrm{Fb}$

for semiconductor charge transport $[12,13]$ and to optical parametric oscillators $[14,15]$.

After such continued theoretical effort, there is still no clear experimental evidence of the existence of this HopfTuring mixed mode [16,17]. We present in this Letter the first neat observation of oscillating spots in a hexagonal lattice using the photosensitive chemical CDIMA (Chlorine dioxide-iodine-malonic acid) reaction [18] in a two-dimensional system. Numerical simulations with the LE model of this reaction confirm the results.

Experiments were performed using the photosensitive CDIMA reaction with chemical concentrations: $\left[\mathrm{I}_{2}\right]_{0}=$ $0.45 \mathrm{mM},\left[\mathrm{ClO}_{2}\right]_{0}=0.1 \mathrm{mM},[\text { Malonic acid }]_{0}=0.9 \mathrm{mM}$, $[\text { Poly }(\text { vinyl alcohol })]_{0}=0.5 \mathrm{~g} / \mathrm{L}$, and $\left[\mathrm{H}_{2} \mathrm{SO}_{4}\right]_{0}=10 \mathrm{mM}$. The setup used is described in Ref. [19].

With these input concentrations, the system is located within the Hopf domain close to the Turing region. A spatially homogeneous and constant in time illumination was used as an additional main control parameter. For low intensity values $\left[I=(2200 \pm 50) \times 10^{-6} \mathrm{~W} / \mathrm{cm}^{2}\right]$, the chemical system gives rise to phase waves of oscillations typical of the Hopf instability. If the illumination is substantially increased the oscillations disappear and steady Turing patterns become stable $[I=(13500 \pm 50) \times$ $\left.10^{-6} \mathrm{~W} / \mathrm{cm}^{2}\right]$. Further increase of the light precludes the formation of any pattern $[I=(20000 \pm 50) \times$ $\left.10^{-6} \mathrm{~W} / \mathrm{cm}^{2}\right]$.

Below the threshold for Turing patterns we have found, however, a window where another type of patterns appears. At a typical external background illumination of intensity $I=(10500 \pm 50) \times 10^{-6} \mathrm{~W} / \mathrm{cm}^{2}$, a pattern composed of spots oscillating in amplitude and hexagonal ordering is observed. Fig. 1(a) is a sequence of successively taken snapshots of the reaction layer displaying the oscillating amplitude of the spots. The spots in hexagonal configuration $(\lambda=0.48 \pm 0.05 \mathrm{~mm})$ oscillate in amplitude and without noticeable position change with a period of $T=$ $4.44 \pm 0.2 \mathrm{~min}$. Oscillations were remarkably stable for 


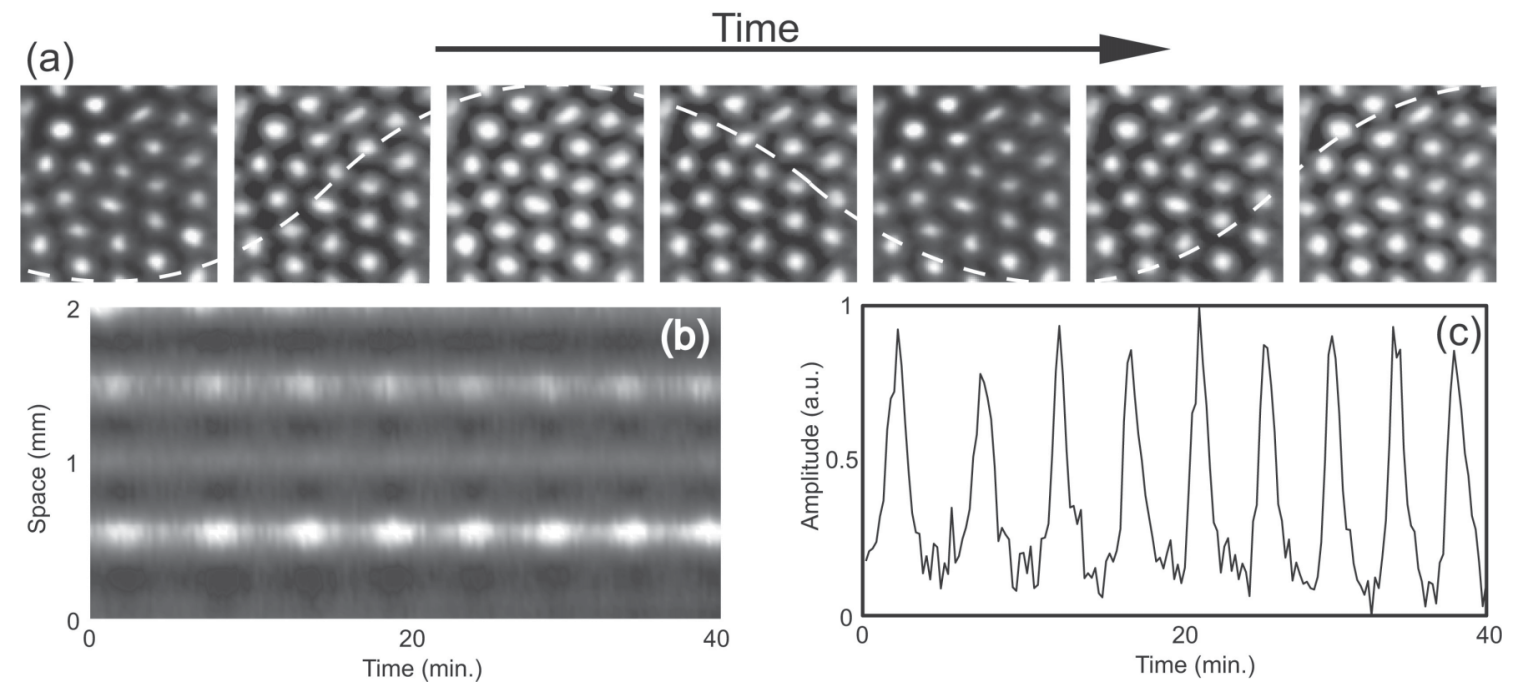

Time

FIG. 1. (a) Snapshots of a region $\left(2.5 \times 2.5 \mathrm{~mm}^{2}\right)$ with oscillating spots in front of time. The dashed sinusoidal line was drawn trying to help the eye to identify the oscillation. (b) Space-time plot of a line of the system in front of time. (c) Local average of the concentration for an area involving around 7 spots.

the whole time of observation (up to $1 \mathrm{~h}$ ) without any significative change in the range of the oscillations, Fig. 1(b). An average over an area containing a few spots, as depicted in Fig. 1(c), provides another signature of the coherent oscillating dynamics. Although some evidence of phase waves of the oscillation have been observed, the medium is not large enough to characterize these phase waves (typical wavelength for phase waves of oscillation under these chemical conditions is around $4 \mathrm{~mm}$, almost 10 times larger than the characteristic distance between spots). Typical Hopf frequency for the CDIMA reaction is around $5 \mathrm{~min}[20,21]$, in the same range that the oscillation in the amplitude of the spots.

The wavelength of the Turing pattern $\lambda$ is larger than the thickness of the gel where the pattern is observed $(0.3 \mathrm{~mm})$. This ensures that there is no three-dimensional or coupled layer effects in the observed pattern and that the structure is indeed effectively two dimensional [17].

Numerical simulations were conducted using the Lengyel-Epstein model for the CDIMA reaction, modified to take into account the light sensitivity $[18,22]$.

$$
\begin{aligned}
& \frac{\partial u}{\partial t}=a-c u-\frac{4 u v}{1+u^{2}}-\phi+\nabla^{2} u, \\
& \frac{\partial v}{\partial t}=\sigma\left(c u-\frac{u v}{1+u^{2}}+\phi+d \nabla^{2} v\right) .
\end{aligned}
$$

Here $u$ and $v$ are the dimensionless concentrations of the activator $\left(\mathrm{I}^{-}\right)$and the inhibitor $\left(\mathrm{ClO}_{2}^{-}\right)$species, whereas $a$, $c$, and $\sigma$ are dimensionless parameters of the chemical system, and $d$ is proportional to the ratio of diffusion coefficients. The parameter $\phi$ plays the role of the illumination intensity. The RD equations were solved using a standard discretization for the Laplacian and a RungeKutta method for the temporal integration. The typical size of the two-dimensional medium was $100 \times$
100 space units ${ }^{2}$. The initial condition is prescribed as a random distribution for both fields around the value of the unstable center of the limit cycle (for the set of parameter values used, see caption of Fig. 2, it corresponds to $u=$ $4 / 3$ and $v=15 / 2$ ). Model parameters conveniently chosen close to the boundary between the Hopf and Turing regimes lead to patterns of oscillating spots, Fig. 2(a), strikingly similar to those observed in experiments. The pattern is composed of a hexagonal array of white spots steady in the space but oscillating in amplitude.

For the parameters values in Fig. 2(a), oscillatory spots with period $T=9.9 \pm 0.1$ time units in hexagonal configuration ( $\lambda=9.5 \pm 0.5$ space units) have been observed in simulations lasting for more than a hundred of oscillations, without noticeable changes on the spot lattice and on the amplitude of the oscillations. As in the experiments, phase waves were observed in large systems $(500 \times$ 500 space units ${ }^{2}$ ). Panels 2(b) and 2(c) are composed similarly to the experimental Fig. 1. Oscillating spots are statistically robust against initial conditions (more than $90 \%$ over 50 randomly chosen realizations of the initial conditions). As another proof of robustness, similar results are observed in a range of values of $\sigma$, between $\sigma=4.1$ and $\sigma=4.5$ keeping the rest of parameters unchanged. Beyond this window the spots are not stable anymore but they either become a transitory solution to Turing patterns or to global oscillations, depending on the parameters. Analogously occurred with variations of $a$.

An alternative parameter analysis concerning the effect of the illumination intensity is shown in Fig. 3. The value of $\phi$ is varied from the null value (not shown), where the numerical system presents global oscillations, to the total elimination of the spatiotemporal patterns $(\phi \sim 3.32)$. For low values of $\phi$, and apart from transients, global oscillations are the preferred solutions, Fig. 3(a). When the 
(a)
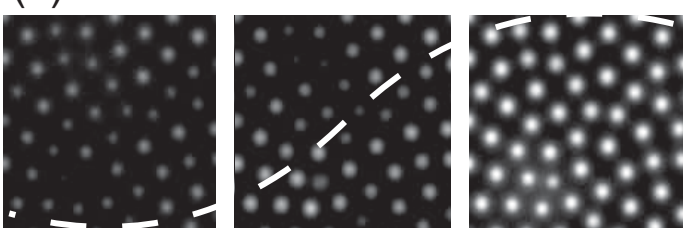

\section{Time}
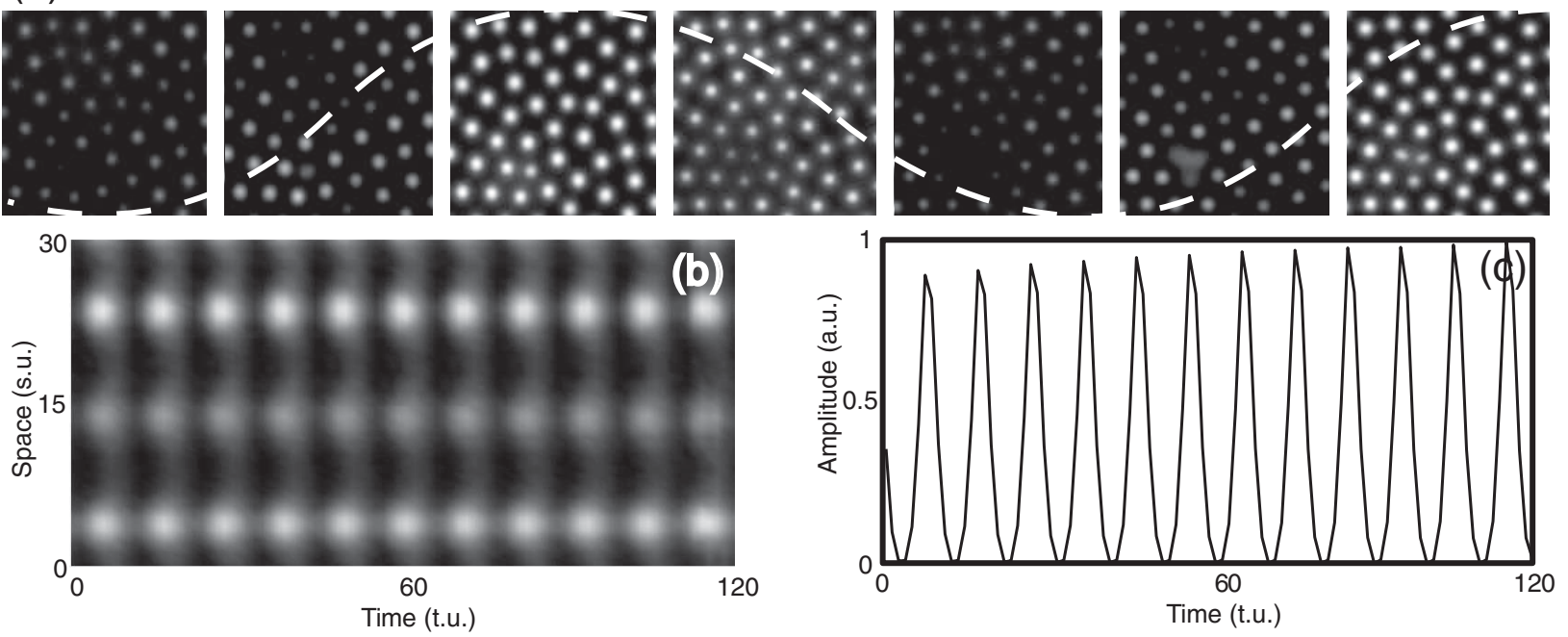

FIG. 2. (a) Snapshots of a region $\left(60 \times 60\right.$ space units $\left.^{2}\right)$ with oscillating spots in front of time. The sinusoidal line helps to identify the oscillation. (b) Space-time plot of a line of the system in front of time. (c) Local average of the concentration for an area involving around 7 spots. The parameter values were: $a=18, c=0.3, \sigma=4.5, d=1.07$, and $\phi=3.20$, with temporal and spatial discretization, respectively, $\Delta t=0.0025$ and $\Delta x=0.5$.

value of $\phi$ is increased there is a gradual lacking of spatial coherence, but the system still oscillates, Fig. 3(b). Further increasing the parameter for illumination, a regime of bistability is crossed, Fig. 3(c), before reaching the mixed mode of oscillating spots, Fig. 3(d), in which we are interested here. It is worth pointing out that, as expected, for large enough systems it is rather common to observe phase waves in the oscillations of the spots as a signature of spatial decorrelation. Further increase of the light stabilizes the spots, and the oscillation is completely suppressed, Fig. 3(e). Finally, above a certain threshold of the parameter $\phi$, spatiotemporal structures are eliminated and the system evolves to a featureless state Fig. 3(f). A numerical phase diagram for the value of the illumination parameter $\phi=3.2$ is shown in Fig. 3(g). There, it can be observed that oscillating spots appear in the vicinity of the cross point between Hopf and Turing lines of the linear stability analysis.

As a small comment let us remark that within the intermediate bistable region of the above parameter range, rather complex solutions can be found corresponding to either coexistence or competition between oscillations and Turing modes, or oscillations versus oscillating spots. These types of dynamic regimes are reminiscent of similar behaviors predicted in Brusselator-like models [7].

In spite of the invested theoretical effort, reviewed in the introductory part of this Letter, the experimental observation of mixed Turing-Hopf modes in the form of oscillating spots, as here reported, had proven elusive in the past. To our understanding we were able to unveil such a phenomenon here by advantageously using illumination as an additional nonequilibrium control parameter of the CDIMA
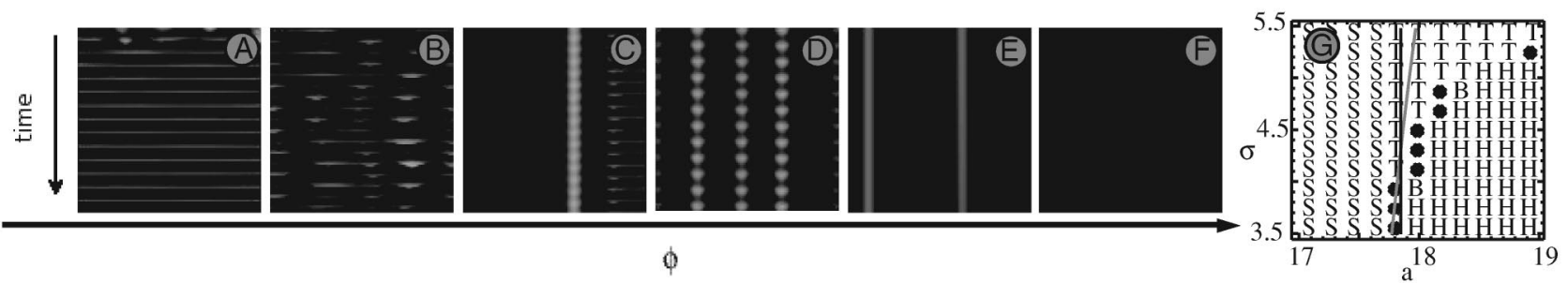

FIG. 3. Numerical space-time plot for different values of the parameter $\phi$ during $t=100$ temporal units in a system of $50 \times$ 50 space units ${ }^{2}$. The dynamics and the values of $\phi$ for the panels showed in the figure correspond to: (A) global oscillations $(\phi=$ $3.15)$, (B) inhomogeneous oscillations $(\phi=3.17)$, (C) bistability $(\phi=3.18)$, (D) oscillatory spots $(\phi=3.21)$, (E) Turing patterns $(\phi=3.25)$, and (F) steady state $(\phi=3.32)$. (G) Numerical phase diagram for $\phi=3.20$. Each point is result of a two-dimensional simulation with random initial conditions: T: Turing, H: Hopf oscillations, S: steady state, B: bistability, O: oscillating spots. Lines corresponds to the stability analysis for the extended system in one dimension. Black line: Turing, gray line: Hopf. The rest of the numerical parameters for all the figures are the same as in Fig. 2. 
reaction. Actually, forcing turns out to be a very convenient strategy to design and control spatiotemporal behavior in chemical systems, as recently reviewed [23]. In particular, light forcing applied to the Turing pattern forming CDIMA reaction has been much investigated during these last years. Either temporal [24], spatial [25], spatiotemporal [26], or even random [27] forcing modes have been considered and the observed patterns interpreted in terms of resonances between the intrinsic and externally imposed spatial and temporal length scales. Contrarily, we tend to believe that, in the situation here, the effect of light forcing is much more instrumental. In fact, CDIMA concentrations to observe contrasted Turing-like patterns, in particular, with reference to poly(vinyl alcohol) (PVA), bring the system well inside the instability. On the other hand, the range of PVA concentrations compatible with reasonably contrasted oscillations is very narrow [28]. This practically precludes the observation of mixed Turing-Hopf modes in the absence of photoactivation. Illumination permits a finer tuning by reducing the medium reactivity and advantageously permitting us to get close enough to the codimension-two bifurcation point with still sufficient contrast. Moreover, in the numerical simulations of the LE model the regions of oscillating spots are observed relatively near to the steady-Turing-Hopf intersection, in agreement with analytic results which predict oscillatory spots near the point where the curves for steady-Turing and steady-Hopf bifurcations cross [7].

In conclusion, in this Letter we have reported experimental evidence of the existence of Turing-Hopf mixed mode in a two-dimensional system. Using the photosensitive CDIMA reaction and homogeneous background illumination as our control parameter, a hexagonal pattern composed of oscillating spots has been observed. Numerical simulations which corroborate the experimental results have been also reported. Our results open the possibility for testing the theoretical aspects predicted for such systems as well as new studies in the field of mixed mode patterns.

Experiments were carried out in Santiago de Compostela. Research was supported by the DGI (Spain) under Projects No. FIS2004-03006 and No. BQU200305042-C02-01, and Xunta de Galicia (Spain) under Project No. PGIDIT05PXIC20607PN. D. G. M. acknowledges financial support from the Ministerio de Educacion y Ciencia in Spain. S. A. acknowledges financial support from E.U. Network "Universal Principles in Nonequilibrium Pattern Formation". We would like to thank Lingfa Yang, Irving R. Epstein, Anne De Wit, and Vladimir K. Vanag for useful discussions and comments.

[1] M. C. Cross and P. C. Hohenberg, Rev. Mod. Phys. 65, 851 (1993).

[2] D. Walgraef, Spatio-Temporal Pattern Formation with Examples in Physics, Chemistry and Materials Science (Springer-Verlag, New York, 1996).
[3] V. Castets, E. Dulos, J. Boissonade, and P. De Kepper, Phys. Rev. Lett. 64, 2953 (1990).

[4] Q. Ouyang and H. L. Swinney, Nature (London) 352, 610 (1991).

[5] A. Rovinsky and M. Menzinger, Phys. Rev. A 46, 6315 (1992).

[6] J. J. Perraud, A. De Wit, E. Dulos, P. De Kepper, G. Dewel, and P. Borckmans, Phys. Rev. Lett. 71, 1272 (1993).

[7] A. De Wit, D. Lima, G. Dewel, and P. Borckmans, Phys. Rev. E 54, 261 (1996).

[8] J. D. Dockery and R. J. Field, Phys. Rev. E 58, 823 (1998).

[9] L. Yang, A. M. Zhabotinsky, and I. R. Epstein, Phys. Rev. Lett. 92, 198303 (2004).

[10] L. Yang, I. Bernstein, and I. R. Epstein, Phys. Rev. Lett. 95, 038303 (2005).

[11] L. Yang and I. R. Epstein, Phys. Rev. Lett. 90, 178303 (2003).

[12] M. Meixner, A. De Wit, S. Bose, and E. Schöll, Phys. Rev. E 55, 6690 (1997).

[13] W. Just, M. Bose, S. Bose, H. Engel, and E. Schöll, Phys. Rev. E 64, 026219 (2001).

[14] M. Tlidi, P. Mandel, and M. Haelterman, Phys. Rev. E 56, 6524 (1997).

[15] P. V. Paulau, I. V. Babushkin, and N.A. Loiko, Phys. Rev. E 70, 046222 (2004).

[16] P. De kepper, J. J. Perraud, B. Rudovics, and E. Dulos, Int. J. Bifurcation Chaos Appl. Sci. Eng. 4, 1215 (1994).

[17] Some evidence of coexistence of Hopf and Turing instability was reported previously by P. De Kepper's group (see Ref. [15]). The gel reactor used was considerably thicker $(3.5 \mathrm{~mm})$ than the one used in our experiments $(0.3 \mathrm{~mm})$, allowing, this way, three-dimensional effects. The reported coexistence was due to the superposition of patterns in different layers of the system subjected to different concentration because of the three dimensionality of the gel. Also, no clear interaction between the two patterns was observed. Therefore, the resulting pattern was considered more an optical superposition than a Turing-Hopf mixed mode.

[18] A. P. Muñuzuri, M. Dolnik, A. M. Zhabotinsky, and I. R. Epstein, J. Am. Chem. Soc. 121, 8065 (1999).

[19] D. G. Míguez, V. Pérez-villar, and A. P. Muñuzuri, Phys. Rev. E 71, 066217 (2005).

[20] D. G. Míguez, R. A. Satnoianu, and A. P. Muñuzuri, Phys. Rev. E 73, 025201(R) (2006).

[21] D. G. Míguez, G. Izús, and A. P. Muñuzuri, Phys. Rev. E 73, 016207 (2006).

[22] I. Lengyel and I. R. Epstein, Science 251, 650 (1991).

[23] A.S. Mikhailov and K. Showalter, Phys. Rep. 425, 79 (2006).

[24] A. K. Horvath, M. Dolnik, A. P. Muñuzuri, A. M. Zhabotinsky, and I. R. Epstein, Phys. Rev. Lett. 83, 2950 (1999).

[25] M. Dolnik, I. Berenstein, A. M. Zhabotinsky, and I. R. Epstein, Phys. Rev. Lett. 87, 238301 (2001).

[26] D. G. Míguez, E. M. Nicola, A.P. Muñuzuri, J. Casademunt, F. Sagués, and L. Kramer, Phys. Rev. Lett. 93, 048303 (2004).

[27] A. Sanz-Anchelergues, A. M. Zhabotinsky, I. R. Epstein, and A. P. Muñuzuri, Phys. Rev. E 63, 056124 (2001).

[28] J. J. Perraud, K. I. Agladze, E. Dulos, and P. De Kepper, Physica (Amsterdam) A188, 1 (1992). 